\title{
IDENTITIES INVOLVING THE COEFFICIENTS OF A CLASS OF DIRICHLET SERIES. I
}

\author{
BY \\ BRUCE C. BERNDT( $\left.{ }^{1}\right)$
}

1. Introduction and preliminary results. Suppose $\phi$ and $\psi$ are Dirichlet series satisfying a functional equation of the form

$$
\Gamma^{m}(s) \phi(s)=\Gamma^{m}(r-s) \psi(r-s),
$$

where $m$ is a positive integer and $r$ is real. For $m=1$, Chandrasekharan and Narasimhan [3] have shown that for a large class of Dirichlet series the functional equation and two types of identities are equivalent. For example, if $\tau(n)$ denotes Ramanujan's arithmetical function and

$$
f(s)=\sum_{n=1}^{\infty} \tau(n) n^{-s}
$$

they showed that

$$
\begin{aligned}
(2 \pi)^{-s} \Gamma(s) f(s) & =(2 \pi)^{-(12-s)} \Gamma(12-s) f(12-s), \\
\sum_{n=1}^{\infty} \tau(n) e^{-n y} & =(2 \pi / y)^{12} \sum_{n=1}^{\infty} \tau(n) e^{-4 \pi^{2} n / y}, \quad y>0,
\end{aligned}
$$

and

$$
\frac{1}{\Gamma(q+1)} \sum_{n \leqq x}^{\prime} \tau(n)(x-n)^{q}=(2 \pi)^{-q} \sum_{n=1}^{\infty} \tau(n)\left(\frac{x}{n}\right)^{6+q / 2} J_{12+q}\left(4 \pi(n x)^{1 / 2}\right)
$$

are equivalent. Here $x>0, q>-\frac{1}{2}, J_{v}(z)$ denotes the usual Bessel function, and the ' indicates that if $n=x, \tau(x)$ is to be multiplied by $\frac{1}{2}$. In this paper we consider the more general case when $m$ is any positive integer and establish the equivalence of relations similar to (1.1), (1.2) and (1.3). We shall examine relations similar to (1.3) in some detail with the aim of establishing such an identity for the least possible $q$. We conclude with some examples.

Throughout the paper we let $s=\sigma+i t$ with $\sigma$ and $t$ both real. If $c$ is real, we denote the integral $\int_{c-i \infty}^{c+i \infty}$ by $\int_{(c)}$. The summation sign $\sum$ appearing with no indices will always mean $\sum_{n=1}^{\infty}$. $A$ always denotes a constant, not necessarily the same with each occurrence.

First, we indicate those Dirichlet series which we are studying.

Received by the editors May 25, 1967 and, in revised form, March 28, 1968.

(1) Some of the results of this paper appeared in the author's Ph.D. dissertation written under the direction of Professor J. R. Smart at the University of Wisconsin in 1966. 
DefinITION 1. Let $\left\{\lambda_{n}\right\}$ and $\left\{\mu_{n}\right\}$ be two sequences of positive numbers tending to $\infty$, and $\{a(n)\}$ and $\{b(n)\}$ two sequences of complex numbers not identically zero. Consider the functions $\phi$ and $\psi$ representable as Dirichlet series

$$
\phi(s)=\sum a(n) \lambda_{n}^{-s}, \quad \psi(s)=\sum b(n) \mu_{n}^{-s}
$$

with finite abscissas of absolute convergence $\sigma_{a}$ and $\sigma_{a}^{*}$, respectively. Let $r$ be real, and let $m$ be a positive integer. We say that $\phi$ and $\psi$ satisfy the functional equation

$$
\Gamma^{m}(s) \phi(s)=\Gamma^{m}(r-s) \psi(r-s)
$$

if there exists in the $s$-plane a domain $D$ which is the exterior of a bounded closed set $S$ such that in $D$ a holomorphic function $\chi(s)$ exists with the properties:

(i) $\chi(s)=\Gamma^{m}(s) \phi(s), \sigma>\sigma_{a} ; \chi(s)=\Gamma^{m}(r-s) \psi(r-s), \sigma<r-\sigma_{a}^{*}$.

(ii) If $\gamma=\sigma_{a}^{*}+p-1 / 4 m$, where $p$ is the least positive integer chosen so that $\gamma>\max \left(0, \sigma_{a}, \sigma_{a}^{*}\right)$, and $S$ lies in the strip $r-\gamma<\sigma<\gamma$, then for some constant $\theta<1$,

$$
\chi(s)=O(\exp [\exp [\theta \pi|s| /(2 \gamma-r)]]),
$$

uniformly in the strip $r-\gamma<\sigma<\gamma,|t| \geqq \eta$, where $\eta$ is chosen so that the strip is contained in $D$.

This definition is similar to that given by Bochner [2] and that given by Chandrasekharan and Narasimhan [3] in the case $m=1$. If $m=1, \gamma a(n)=b(n)$ with $\gamma= \pm 1, r>0, \lambda_{n}=\mu_{n}=2 \pi n / \lambda$ with $\lambda>0$, and $(s-r) \phi(s)$ is entire, then $(2 \pi / \lambda)^{s} \phi(s)$ is a Dirichlet series of signature $(\lambda, r, \gamma)$ according to the definition of Hecke [7]. Thus, $f(s)$ is of signature $(1,12,1)$, and $\zeta(2 s)$ is of signature $(2,1 / 2,1)$.

We next give Bochner's definition of a residual function [2].

Definition 2. $P$ is said to be a residual function if:

(i) $P$ is defined and differentiable on $(0, \infty)$.

(ii) $P(x)=O\left(x^{-c}\right)$ as $x \rightarrow 0$, and $P(x)=O\left(x^{c}\right)$ as $x \rightarrow \infty$, for some constant $c>0$, so that the functions

$$
I_{r}(s)=\int_{0}^{1} P(x) x^{s-1} d x, \quad I_{l}(s)=-\int_{1}^{\infty} P(x) x^{s-1} d x
$$

can be introduced in a right half-plane and a left half-plane, respectively.

(iii) $I_{r}$ and $I_{l}$ can be analytically continued into each other in a domain $D$ as given in definition 1.

(iv) $\lim _{|t| \rightarrow \infty} I(\sigma+i t)=0$, uniformly in $-\infty<\sigma_{1} \leqq \sigma \leqq \sigma_{2}<\infty$, where $I$ denotes the function obtained by analytic continuation in (iii).

We shall need several preliminary lemmas.

LEMMA 1. If $\chi(s)$ is analytic in a domain $D$ of the s-plane, then any integral

$$
P(x)=\frac{1}{2 \pi i} \int_{C} \chi(s) x^{-s} d s
$$

over a bounded curve or curves in $D$, with $x^{-s}=\exp [-s \log x]$, is a residual function. Conversely, any residual function can be so represented. 
Lemma 1 is due to Bochner [2].

LemMA 2. Let $f$ be holomorphic in a strip $S$ given by $a<\sigma<b,|t|>\eta>0$, and continuous on the boundary. If for some constant $\theta<1$,

$$
f(s)=O(\exp [\exp [\theta \pi|s| /(b-a)]]),
$$

uniformly in $S, f(a+i t)=o(1)$ and $f(b+i t)=o(1)$ as $|t| \rightarrow \infty$, then $f(\sigma+i t)=o(1)$ uniformly in $S$ as $|t| \rightarrow \infty$.

This is a version of the Phragmén-Lindelöf theorem [8, p. 109].

LEMMA 3. We have

$$
|\Gamma(s)| \sim(2 \pi)^{1 / 2}|t|^{\sigma-1 / 2} e^{-(1 / 2) \pi|t|},
$$

for fixed $\sigma$, as $|t|$ tends to $\infty$.

A proof of Lemma 3 may be found in [6, p. 224].

Lemma 4. Let $\phi(s)=\sum a(n) \lambda_{n}^{-s}$. Then, for $c>\max \left(0, \sigma_{a}\right)$ and $q \geqq 0$,

$$
\frac{1}{\Gamma(q+1)} \sum_{\lambda_{n} \leqq x}^{\prime} a(n)\left(x-\lambda_{n}\right)^{q}=\frac{1}{2 \pi i} \int_{(c)} \frac{\Gamma(s) \phi(s) x^{s+q}}{\Gamma(s+q+1)} d s .
$$

This result is given in [3].

DefinITION 3. Let $x, c>0$ and suppose $m$ is a positive integer. Define

$$
E_{m}(x)=\frac{1}{2 \pi i} \int_{(c)} \Gamma^{m}(s) x^{-s} d s .
$$

As a consequence of $E_{1}(x)=e^{-x}[11, \mathrm{p} .7]$, and a general theorem on multiple Mellin transforms [11, pp. 53, 60], we have

Lemma 5. For $x, c>0$,

$$
E_{m}(x)=\int_{0}^{\infty} e^{-u_{m-1}} \frac{d u_{m-1}}{u_{m-1}} \int_{0}^{\infty} e^{-u_{m-2}} \frac{d u_{m-2}}{u_{m-2}} \cdots \int_{0}^{\infty} \exp \left[-u_{1}-x / u_{1} \cdots u_{m-1}\right] \frac{d u_{1}}{u_{1}}
$$

LEMMA 6. We have

$$
E_{m}(x)=O\left(\exp \left[-x^{1 / m}\right]\right)
$$

as $x \rightarrow \infty$.

Proof. We proceed by induction. The result is trivial for $m=1$. Assuming the result is valid for $E_{m-1}$, we have by Lemma 5 for $m \geqq 2$,

$$
E_{m}(x)=O\left(\int_{0}^{\infty} \exp \left[-u-(x / u)^{1 /(m-1)}\right] \frac{d u}{u}\right) .
$$

Write this integral as

$$
\left(\int_{0}^{1}+\int_{1}^{\infty}\right) \exp \left[-x^{1 / m}\left(u+u^{-1 /(m-1)}\right)\right] \frac{d u}{u}=I_{1}+I_{2} .
$$


Now $f(u)=\exp \left\{-x^{1 / m} u^{-1 /(m-1)}\right\} / u$ obtains its maximum value when

$$
u=\frac{x^{(m-1) / m}}{(m-1)^{m-1}}
$$

which we may assume is greater than 1 without loss of generality. Since $f(u)$ is then increasing on $(0,1)$,

$$
I_{1} \leqq \exp \left[-x^{1 / m}\right] \int_{0}^{1} \exp \left[-x^{1 / m} u\right] d u=O\left(\exp \left[-x^{1 / m}\right]\right)
$$

Secondly,

$$
I_{2} \leqq A x^{-(m-1) ! m} \int_{1}^{\infty} \exp \left[-x^{1 / m} u\right] d u=O\left(\exp \left(-x^{1 / m}\right]\right)
$$

Lemma 7. For $0<c<\nu / 2+3 / 4$,

$$
J_{v}(x)=\frac{1}{2 \pi i} \int_{(c)} \frac{\Gamma(s)}{\Gamma(\nu+1-s)}\left(\frac{x}{2}\right)^{v-2 s} d s .
$$

This formula is given in [11, formula (7.9.1), p. 196].

Definition 4. For any positive integer $m \geqq 2$ define

$$
\begin{aligned}
K_{v}(x ; \mu ; m)=\int_{0}^{\infty} u_{m-1}^{v-\mu-1} J_{\mu}\left(u_{m-1}\right) d u_{m-1} & \int_{0}^{\infty} u_{m-2}^{v-\mu-1} J_{\mu}\left(u_{m-2}\right) d u_{m-2} \\
& \cdots \int_{0}^{\infty} u_{1}^{\nu-\mu-1} J_{\mu}\left(u_{1}\right) J_{v}\left(x / u_{1} \cdots u_{m-1}\right) d u_{1},
\end{aligned}
$$

provided that $\mu, \nu>-3 / 2$ so that the integral converges. We extend this definition to $m=1$ by taking $K_{v}(x ; \mu ; 1)=J_{v}(x)$.

LEMMA 8. For $0<c<\min (\mu, v) / 2+3 / 4$ and $m \geqq 1$,

$$
x^{-v} K_{v}(x ; \mu ; m)=\frac{1}{2 \pi i} \int_{(c)} \frac{2^{2 m s-(m-1) \mu-v-m+1} \Gamma^{m}(s) x^{-2 s}}{\Gamma^{m-1}(\mu+1-s) \Gamma(\nu+1-s)} d s .
$$

Lemma 8 is a consequence of Lemma 7 and the aforementioned theorem on multiple Mellin transforms.

Using the definition of $K_{v}(x ; \mu ; m)$ and the fact that

$$
(d / d x)\left[x^{v} J_{v}(x)\right]=x^{v} J_{v-1}(x)
$$

$[13$, p. 45], we immediately have

Lemma 9.

$$
(d / d x)\left[x^{v} K_{v}(x ; \mu ; m)\right]=x^{v} K_{v-1}(x ; \mu ; m) .
$$

From Lemma 8 and a result of Chandrasekharan and Narasimhan [5, Lemma 1] we may deduce 
LEMMA 10. For $m \geqq 1$ and $\min (r-1, v)+3 / 2>0$,

$$
\begin{aligned}
K_{v}(x ; r-1 ; m) \sim x^{(1-1 / m)(v-r)-1 / 2 m}\left(\exp \left[i m x^{1 / m}\right] \sum_{n=0}^{\infty}\right. & d_{n} x^{-n / m} \\
& \left.+\exp \left[-i m x^{1 / m}\right] \sum_{n=0}^{\infty} d_{n}^{\prime} x^{-n / m}\right),
\end{aligned}
$$

as $x \rightarrow \infty$, where $d_{n}$ and $d_{n}^{\prime}$ are constants, $n=0,1, \ldots$

LeMma 11. As $x \rightarrow 0, K_{v}(x ; \mu ; m)=O\left(x^{\nu}\right)$.

This result is a consequence of the fact that $J_{v}(x)=O\left(x^{v}\right)$ as $x \rightarrow 0$.

2. The equivalence of three identities.

THEOREM 1. Let $y>0 . \phi$ and $\psi$ satisfy functional equation (1.4) if and only if

$$
\sum a(n) E_{m}\left(\lambda_{n} y\right)=y^{-r} \sum b(n) E_{m}\left(\mu_{n} / y\right)+P(y),
$$

where $P(y)$ is a residual function given by

$$
P(y)=\frac{1}{2 \pi i} \int_{C} \chi(s) y^{-s} d s,
$$

where $C$ denotes a curve or curves containing $S$.

A proof of Theorem 1 has been indicated by Bochner [2].

Proof. If $c>\max \left(0, \sigma_{a}, \sigma_{a}^{*}\right)$, we easily deduce from the definition of $E_{m}(x)$ that

$$
\begin{aligned}
\sum a(n) E_{m}\left(\lambda_{n} y\right) & =\sum a(n) \frac{1}{2 \pi i} \int_{(c)} \Gamma^{m}(s)\left(\lambda_{n} y\right)^{-s} d s \\
& =\frac{1}{2 \pi i} \int_{(c)} \Gamma^{m}(s) \phi(s) y^{-s} d s,
\end{aligned}
$$

where we have inverted the order of summation and integration by absolute convergence. We suppose $c$ is large enough so that $C$ is located within the strip $r-c<\sigma<c$. Consider now the integrand on the right-hand side of (2.2) and integrate over the rectangle with vertices $c \pm i T$ and $c-r \pm i T$. By Lemmas 2 and 3 the integrals on the horizontal edges tend to 0 as $T$ tends to $\infty$. Thus, letting $T$ tend to $\infty$, applying the residue theorem, and using the functional equation, we have

$$
\begin{aligned}
\frac{1}{2 \pi i} \int_{(c)} \Gamma^{m}(s) \phi(s) y^{-s} d s & =\frac{1}{2 \pi i} \int_{(r-c)} \Gamma^{m}(r-s) \psi(r-s) y^{-s} d s+P(y) \\
& =\frac{y^{-r}}{2 \pi i} \int_{(c)} \Gamma^{m}(s) \psi(s)(1 / y)^{-s} d s+P(y) \\
& =y^{-r} \sum b(n) E_{m}\left(\mu_{n} / y\right)+P(y),
\end{aligned}
$$

where we have again inverted the order of summation and integration by absolute convergence. 
We now prove the converse. By Mellin's inversion formula for $\sigma>0$,

$$
\Gamma^{m}(s)=\int_{0}^{\infty} x^{s-1} E_{m}(x) d x
$$

from which it follows that

$$
\Gamma^{m}(s) \phi(s)=\sum a(n) \int_{0}^{\infty} x^{s-1} E_{m}\left(\lambda_{n} x\right) d x .
$$

Inverting the order of summation and integration with the aid of Lemma 6, letting

$$
\Phi(x)=\sum a(n) E_{m}\left(\lambda_{n} x\right), \quad \Psi(x)=\sum b(n) E_{m}\left(\mu_{n} x\right),
$$

and using (2.1), we have from (2.3)

$$
\begin{aligned}
\Gamma^{m}(s) \phi(s) & =\int_{0}^{1} x^{s-1} \Phi(x) d x+\int_{1}^{\infty} x^{s-1} \Phi(x) d x \\
& =\int_{0}^{1} x^{s-r-1} \Psi(1 / x) d x+\int_{0}^{1} x^{s-1} P(x) d x+\int_{1}^{\infty} x^{s-1} \Phi(x) d x \\
& =\int_{1}^{\infty}\left(x^{s-1} \Phi(x)+x^{r-s-1} \Psi(x)\right) d x+\int_{0}^{1} x^{s-1} P(x) d x
\end{aligned}
$$

Now, as $x \rightarrow \infty$,

$$
\Phi(x)=O\left(\exp \left[-\lambda_{j} x^{1 / m}\right]\right), \quad \Psi(x)=O\left(\exp \left[-\mu_{k} x^{1 / m}\right]\right),
$$

where $a(n)=0,0<n<j, a(j) \neq 0$, and $b(n)=0,0<n<k, b(k) \neq 0$. Hence, the first integral on the right-hand side of $(2.4)$ by analytic continuation represents an entire function.

In a like manner we have

$$
\begin{aligned}
\Gamma^{m}(s) \psi(s) & =\int_{0}^{\infty} x^{s-1} \Psi(x) d x \\
& =\int_{0}^{1} x^{s-r-1} \Phi(1 / x) d x-\int_{0}^{1} x^{s-r-1} P(1 / x) d x+\int_{1}^{\infty} x^{s-1} \Psi(x) d x \\
& =\int_{1}^{\infty}\left(x^{s-1} \Psi(x)+x^{r-s-1} \Phi(x)\right) d x-\int_{1}^{\infty} x^{r-s-1} P(x) d x
\end{aligned}
$$

As before, the first term on the right-hand side of (2.6) represents an entire function. Upon the replacement of $s$ by $r-s$, (1.4) easily follows from the hypothesis that $I_{r}(s)$ and $I_{l}(s)$ are equal upon analytic continuation into each other in a domain $D$ as given in Definition 1. We have still to show (1.5). For the second integrals on the right-hand sides of (2.4) and (2.6), (1.5) is obvious from Definition 2. It is then sufficient to examine

$$
\int_{1}^{\infty}\left(x^{s-1} \Phi(x)+x^{r-s-1} \Psi(x)\right) d x=\int_{0}^{\infty}\left(e^{(\sigma+i t) x} \Phi\left(e^{x}\right)+e^{\left.(r-\sigma-i t) \Psi\left(e^{x}\right)\right) d x} .\right.
$$


By (2.5) and the Riemann-Lebesgue lemma this integral tends to 0 as $t$ tends to $\infty$, uniformly in any strip $-\infty<\sigma_{1} \leqq \sigma \leqq \sigma_{2}<\infty$. This completes the proof of Theorem 1 .

THEOREM 2. Let $\phi$ and $\psi$ satisfy functional equation (1.4). If $m \geqq 2$, suppose $r>-1 / 2$. If $x>0$ and $q>2 m \sigma_{a}^{*}-m r-1 / 2$,

$$
\begin{aligned}
& \frac{1}{\Gamma(q+1)} \sum_{\lambda_{n} \leqq x}^{\prime} a(n)\left(x-\lambda_{n}\right)^{q} \\
& \quad=2^{q(1-m)} \sum b(n)\left(\frac{x}{\mu_{n}}\right)^{(r+q) / 2} K_{r+q}\left(2^{m}\left(\mu_{n} x\right)^{1 / 2} ; r-1 ; m\right)+Q_{q}(x),
\end{aligned}
$$

where the series on the right-hand side is absolutely convergent, and $Q_{q}(x)$ is a residual function given by

$$
Q_{q}(x)=\frac{1}{2 \pi i} \int_{C} \frac{\Gamma(s) \phi(s) x^{s+q}}{\Gamma(s+q+1)} d s
$$

where $C$ is a curve or curves encircling all of $S$. Conversely, (2.7) implies functional equation (1.4).

Proof. Let $\gamma$ be as given in Definition 1. By Lemma 4 for $q \geqq 0$,

$$
\frac{1}{2 \pi i} \int_{(\gamma)} \frac{\Gamma(s) \phi(s) x^{s+q}}{\Gamma(s+q+1)} d s=\frac{1}{\Gamma(q+1)} \sum_{\lambda_{n} \leqq x}^{\prime} a(n)\left(x-\lambda_{n}\right)^{q} .
$$

We now move the line of integration to $r-\gamma+i t,-\infty<t<\infty$, by integrating around the rectangle with vertices $\gamma \pm i T, r-\gamma \pm i T$ and then letting $T$ tend to $\infty$. $T$ is chosen large enough so that all of $S$ is contained within the rectangle. Since $\gamma>\sigma_{a}, \phi(\gamma+i t)=O(1)$ as $|t| \rightarrow \infty$. Hence, by Lemma 3 for $s=\gamma+i t$,

$$
\Gamma(s) \phi(s) / \Gamma(s+q+1)=O\left(|t|^{-q-1}\right)=o(1)
$$

as $|t| \rightarrow \infty$, since $q \geqq 0$. Since $\gamma>\sigma_{a}^{*}, \psi(\gamma+i t)=O(1)$ as $|t| \rightarrow \infty$. Then, upon using the functional equation and Lemma 3 , we find for $s=r-\gamma+i t$

$$
\frac{\Gamma(s) \phi(s)}{\Gamma(s+q+1)}=\frac{\Gamma^{m}(r-s) \psi(r-s)}{\Gamma^{m-1}(s) \Gamma(s+q+1)}=O\left(|t|^{2 m \gamma-m r-q-1}\right)=o(1),
$$

as $|t| \rightarrow \infty$, provided $q>2 m \gamma-m r-1$. It now follows from Lemma 2 that

$$
\frac{\Gamma(s) \phi(s)}{\Gamma(s+q+1)}=\frac{\chi(s)}{\Gamma^{m-1}(s) \Gamma(s+q+1)}=o(1),
$$

as $|t| \rightarrow \infty$, uniformly in the strip $r-\gamma \leqq \sigma \leqq \gamma$. Hence, the integrals along the two horizontal sides of the rectangle tend to 0 as $T \rightarrow \infty$, and we have shown that

$$
\frac{1}{2 \pi i} \int_{(y)} \frac{\Gamma(s) \phi(s) x^{s+q}}{\Gamma(s+q+1)} d s=\frac{1}{2 \pi i} \int_{(r-\gamma)} \frac{\Gamma(s) \phi(s) x^{s+q}}{\Gamma(s+q+1)} d s+Q_{q}(x)
$$

provided $q>2 m \gamma-m r-1$. 
Let $I(x)$ denote the first term on the right-hand side of (2.9). Replacing $s$ by $r-s$, using the functional equation, and inverting the order of summation and integration, we find

$$
I(x)=x^{r+q} \sum b(n) \frac{1}{2 \pi i} \int_{(y)} \frac{\Gamma^{m}(s)\left(\mu_{n} x\right)^{-s}}{\Gamma^{m-1}(r-s) \Gamma(r+q+1-s)} d s,
$$

provided $q>2 m \gamma-m r$. By Lemma 8 we have

$$
x^{-r-q} K_{r+q}(x ; r-1 ; m)=\frac{1}{2 \pi i} \int_{(c)} \frac{2^{2 m s-m r-q} \Gamma^{m}(s) x^{-2 s}}{\Gamma^{m-1}(r-s) \Gamma(r+q+1-s)} d s,
$$

provided $0<c<(r-1) / 2+3 / 4$ if $m \geqq 2$, and $0<c<(r+q) / 2+3 / 4$ if $m=1$. However, by Lemma 3 and Cauchy's theorem we can move the line of integration to $\gamma+i t$, $-\infty<t<\infty$, where $q>2 m \gamma-m r$. Thus, (2.10) is valid provided $q>2 m \gamma-m r$ and $r>-\frac{1}{2}$ if $m \geqq 2$. Hence, using (2.10) we conclude

$$
I(x)=2^{q(1-m)} \sum b(n)\left(\frac{x}{\mu_{n}}\right)^{(r+q) / 2} K_{r+q}\left(2^{m}\left(\mu_{n} x\right)^{1 / 2} ; r-1 ; m\right) .
$$

Combining (2.8), (2.9) and (2.11), we have shown (2.7) provided $q \geqq 0$ and $q>2 m \gamma-m r$.

Now, by Lemma 10 ,

$$
\begin{aligned}
I(x) & =O\left(x^{r / 2+q(1-1 / 2 m)-1 / 4 m} \sum|b(n)| \mu_{n}^{-(r / 2+q / 2 m+1 / 4 m)}\right) \\
& =O\left(x^{r / 2+q(1-1 / 2 m)-1 / 4 m}\right),
\end{aligned}
$$

provided $q>2 m \sigma_{a}^{*}-m r-\frac{1}{2}$. Thus, the series on the right-hand side of (2.7) converges absolutely for $q>2 m \sigma_{a}^{*}-m r-\frac{1}{2}$. By $2 m p$ differentiations with the aid of Lemma 9, identity (2.7) may be then upheld for $q>2 m \sigma_{a}^{*}-m r-\frac{1}{2}$ and $q \geqq 0$. However, the uniform and absolute convergence for $q>2 m \sigma_{a}^{*}-m r-\frac{1}{2}$ implies that the $2 m p$ th derivative is continuous. Hence, the left side of (2.7) is continuous for $q>2 m \sigma_{a}^{*}$ $-m r-\frac{1}{2}$, which implies that $q>0$, since the left side of (2.7) is not continuous on $(0, \infty)$ for $q \leqq 0$.

To prove that (2.7) implies (1.4), it is sufficient from Theorem 1 to show that (2.7) implies (2.1).

Multiply both sides of (2.7) by

$$
\begin{aligned}
y^{q+1} \int_{0}^{\infty} \exp \left[-u_{m-1}\right] \frac{d u_{m-1}}{u_{m-1}^{q+2}} \int_{0}^{\infty} \exp \left[-u_{m-2}\right] \frac{d u_{m-2}}{u_{m-2}^{q+2}} \\
\cdots \int_{0}^{\infty} \exp \left[-u_{1}-x y / u_{1} \cdots u_{m-1}\right] \frac{d u_{1}}{u_{1}^{q+2}}
\end{aligned}
$$

and integrate with respect to $x$ over $(0, \infty)$. We assume $q$ is large enough so that all operations are valid. After a routine calculation the left-hand side of (2.7) then yields $\sum a(n) E\left(\lambda_{n} y\right)$ with the aid of Lemma 5. Another easy calculation shows that the second term on the right-hand side of (2.7) yields $P(y)$. After several 
changes in the order of integration and the use of Lemma 8, the first term on the right-hand side of (2.7) gives $y^{-r} \sum b(n) E\left(\mu_{n} / y\right)$, and the proof is complete.

Combining Theorems 1 and 2, we have

THEOREM 3. Functional equation (1.4), the "modular" relation (2.1), and identity (2.7) are equivalent.

We now state some corollaries of Theorem 2 .

CoRollary 1. Suppose $f(s)=\sum a(n) n^{-s}$ is a Dirichlet series of signature $(\lambda, r, \gamma)$. Let $\rho$ denote the residue of $f$ at $s=r$ and $\rho$ ' the constant term in the Laurent expansion of $f$ about $s=r$. Let $\psi(s)=\Gamma^{\prime}(s) / \Gamma(s)$. Then, for $x>0$ and $q>4 \sigma_{a}-2 r-\frac{1}{2}$,

$$
\begin{aligned}
& \frac{1}{\Gamma(q+1)} \sum_{0<m n \leqq x}^{\prime} a(m) a(n)(x-m n)^{q} \\
&=\frac{\phi^{2}(0) x^{q}}{\Gamma(q+1)}+\frac{\Gamma(r) \rho^{2} x^{r+q}}{\Gamma(r+q+1)}\left[\log x+\psi(r)-\psi(r+q+1)+2 \rho^{\prime} / \rho\right] \\
&+\left(\frac{\lambda^{2}}{8 \pi^{2}}\right)^{q} \sum a^{\prime}(n)\left(\frac{x}{n}\right)^{(r+q) / 2} K_{r+q}\left(16 \pi^{2}(n x)^{1 / 2} / \lambda^{2} ; r-1 ; 2\right)
\end{aligned}
$$

where

$$
a^{\prime}(n)=\sum_{j k=n} a(j) a(k),
$$

and where the series on the right-hand side converges absolutely.

Corollary 1 is a consequence of (2.7) and the properties of $f(s)$ stated prior to Definition 1.

Corollary 2. For $x>0$ and $q>2 m \sigma_{a}^{*}-m r-1 / 2$,

$$
\frac{1}{\Gamma(q+1)} \sum_{\lambda_{n} \leqq x} a(n)\left(x-\lambda_{n}\right)^{q}=Q_{q}(x)+O\left(x^{r / 2+q(1-1 / 2 m)-1 / 4 m}\right) .
$$

This result is immediate from (2.12).

$O$-estimates for the sum of Corollary 2 have been given by Richert [10] for a large class of Dirichlet series which overlaps with our class. However, if $\phi \neq \psi$, Richert's estimates are complicated by the need for estimates for $a(n)$. If $\phi=\psi$ and $r \geqq 0$, Corollary 2 is an improvement by a factor of $x^{\varepsilon}, \varepsilon>0$.

The critical strip for $\phi(s)$ is that vertical strip defined by $r-\sigma_{a} \leqq \sigma \leqq \sigma_{a}$. The width is $2 \sigma_{a}-r$.

COROllary 3. The width of the critical strip is at least $1 / 2 m$.

Proof. In the proof of Theorem 2 we saw that the condition $q>2 m \sigma_{a}^{*}-m r-\frac{1}{2}$ implies that $q>0$. Thus, $2 m \sigma_{a}^{*}-m r-\frac{1}{2} \geqq 0$, or $2 \sigma_{a}^{*}-r \geqq 1 / 2 m$.

Apostol and Sklar [1] first noted Corollary 3 in the case $m=1$. For $m=1$ this result is the best possible, for the width of the critical strip for $\zeta(2 s)$ is exactly $1 / 2$. 
3. A theorem on the validity of (2.7). We now wish to establish (2.7) for the lowest possible value of $q$. The methods to be employed are exactly those developed by Chandrasekharan and Narasimhan [3] in the examination of (2.7) for $m=1$. These methods, in turn, are based on results of Zygmund [14] for equiconvergent series.

DEFINITION 5. $\sum_{-\infty}^{\infty} a_{j}(x)$ and $\sum_{-\infty}^{\infty} b_{j}(x)$ are uniformly equiconvergent on an interval if $\sum_{-n}^{n}\left[a_{j}(x)-b,(x)\right]$ converges uniformly on that interval as $n \rightarrow \infty$.

The following two lemmas proven by Chandrasekharan and Narasimhan are of central importance [3, pp. 11, 12].

LEMMA 12. Let $\left\{\alpha_{n}\right\}$ be a positive sequence of numbers tending to $\infty$, and suppose that $\alpha_{-n}=\alpha_{n}$. Suppose that $J$ is a closed interval contained in an interval I of length $2 \pi$. Let $\lambda$ be a $C^{\infty}$ function with compact support on I and which is equal to 1 on J. Assume

$$
\sum_{-\infty}^{\infty}|c(n)|<\infty
$$

Then, if $g$ is a function with period $2 \pi$ which equals $\sum_{-\infty}^{\infty} c(n) \exp \left[i \alpha_{n} x\right]$ on $I$, the Fourier series of $g$ converges uniformly on $J$.

LEMMA 13. With the same notation as Lemma 12 assume

$$
\sup _{0 \leqq h \leqq 1}\left|\sum_{k<a_{n}<k+h} c(n)\right|=o(1)
$$

as $k \rightarrow \infty$, and

$$
\sum_{-\infty}^{\infty}|c(n)| \alpha_{n}^{-1}<\infty
$$

Furthermore, let $\gamma$ be a $C^{\infty}$ function. Then, the series $\gamma(x) \sum_{-\infty}^{\infty} c(n) \exp \left[i \alpha_{n} x\right]$ is uniformly equiconvergent on $J$ with the differentiated series of the Fourier series of a function with period $2 \pi$ which equals $\lambda(x) \sum_{-\infty}^{\infty} c(n) W_{n}(x)$ on $I$, where $W_{n}(x)$ is an antiderivative of $\gamma(x) \exp \left[i \alpha_{n} x\right]$.

Lemma 14. Let $\{b(n)\}$ and $\left\{\mu_{n}\right\}$ be given as in Definition 1. Suppose that

$$
\sum|b(n)| \mu_{n}^{-[r(m-1)+v+3 / 2] / 2 m}<\infty
$$

and

$$
\sup _{0 \leqq h \leqq 1}\left|\sum_{k^{2 m} \leqq \mu_{n} \leqq(k+h)^{2 m}} b(n) \mu_{n}^{-[r(m-1)+v+1 / 2] / 2 m}\right|=o(1)
$$

as $k \rightarrow \infty$. Define for $y>0$,

$$
F_{v}(y)=\sum b(n)\left(\frac{y^{2 m}}{\mu_{n}}\right)^{v / 2} K_{v}\left(2^{m} \mu_{n}^{1 / 2} y^{m} ; r-1 ; m\right) .
$$

Then, $m 2^{m} y^{2 m-1} F_{v}(y)$ is uniformly equiconvergent on any interval $J$ of length less 
than $\pi / m$ with the differentiated series of the Fourier series of a function with period $\pi / m$ which on I equals $\lambda(y) F_{v+1}(y)$, where $I$ is of length $\pi / m$ and contains $J$.

Proof. Examine the function

$$
\begin{aligned}
f(y)=m 2^{m} y^{2 m-1}+m v & \sum b(n) \mu_{n}^{-v / 2}\left\{K_{v}\left(2^{m} \mu_{n}^{1 / 2} y^{m} ; r-1 ; m\right)\right. \\
& -\left(\mu_{n}^{1 / 2} y^{m}\right)^{(1-1 / m)(v-r)-1 / 2 m}\left[d_{0} \exp \left[m i \mu_{n}^{1 / 2 m} y\right]+d_{0}^{\prime} \exp \left[-m i \mu_{n}^{1 / 2 m} y\right]\right] \\
& \left.-\left(\mu_{n}^{1 / 2} y^{m}\right)^{(1-1 / m)(v-r)-3 / 2 m}\left[d_{1} \exp \left[m i \mu_{n}^{1 / 2 m} y\right]+d_{1}^{\prime} \exp \left[-m i \mu_{n}^{1 / 2 m} y\right]\right]\right\}
\end{aligned}
$$

for $y>0$. By Lemma 10 and (3.1) we find

$$
\left|f^{\prime}(y)\right| \leqq A y^{c} \sum|b(n)| \mu_{n}^{-[r(m-1)+v+3 / 2] / 2 m}<\infty
$$

where $c$ is a constant. Hence, $f$ is continuously differentiable for $y>0$. Let $g$ be a function with period $\pi / m$ which equals $f$ on $I$. Since $f$ is continuously differentiable, the Fourier series of $g$ is uniformly convergent on $J$.

Now consider

$$
\begin{aligned}
& m 2^{m} y^{2 m-1} \sum b(n)\left(\frac{y^{2 m}}{\mu_{n}}\right)^{v / 2}\left(\mu_{n}^{1 / 2} y^{m}\right)^{(1-1 / m)(v-r)-1 / 2 m} \\
& \cdot\left[d_{0} \exp \left[m i \mu_{n}^{1 / 2 m} y\right]+d_{0}^{\prime} \exp \left[-m i \mu_{n}^{1 / 2 m} y\right]\right] .
\end{aligned}
$$

In view of (3.1) and (3.2) the hypotheses of Lemma 13 are satisfied with $c(n)$ $=b(n) \mu_{n}^{-[r(m-1)+v+1 / 2] / 2 m}$ and $\alpha_{n}=\mu_{n}^{1 / 2 m}$. Hence, (3.3) is uniformly equiconvergent on $J$ with the differentiated series of the Fourier series of a function with period $\pi / m$, which equals on $I$

$m 2^{m} \lambda(y) \sum b(n) \int_{\alpha}^{y} t^{2 m-1}\left(\frac{t^{2 m}}{\mu_{n}}\right)^{v / 2}\left(\mu_{n}^{1 / 2} t^{m}\right)^{(1-1 / m)(v-r)-1 / 2 m}$

$\cdot\left[d_{0} \exp \left[m i \mu_{n}^{1 / 2 m} t\right]+d_{0}^{\prime} \exp \left[-m i \mu_{n}^{1 / 2 m} t\right]\right] d t$,

where $\alpha>0$.

Next, we examine

$$
\begin{aligned}
m 2^{m} y^{2 m-1} \sum b(n)\left(\frac{y^{2 m}}{\mu_{n}}\right)^{v / 2}\left(\mu_{n}^{1 / 2} y^{m}\right)^{(1-1 / m)(v-r)-3 / 2 m} \\
\cdot\left[d_{1} \exp \left[m i \mu_{n}^{1 / 2 m} y\right]+d_{1}^{\prime} \exp \left[-m i \mu_{n}^{1 / 2 m} y\right]\right] .
\end{aligned}
$$

By (3.1), (3.2) and Lemma 12 with $c(n)=b(n) \mu_{n}^{-[r(m-1)+v+3 / 2] / 2 m}$, the Fourier series of the function with period $\pi / m$ which equals (3.4) on $I$ converges uniformly on $J$.

Thus, it follows that $m 2^{m} y^{2 m-1} F_{v}(y)$ is uniformly equiconvergent on $J$ with the differentiated series of the Fourier series of a function with period $\pi / m$ which on $I$ equals

$$
\lambda(y) \sum b(n) \mu_{n}^{-v / 2} \int_{\alpha_{n}}^{y} m 2^{m} t^{2 m-1+m v} K_{v}\left(2^{m} \mu_{n}^{1 / 2} t^{m} ; r-1 ; m\right) d t=\lambda(y) F_{v+1}(y),
$$

where we have employed Lemma 9 and have chosen $\alpha_{n}>0$ so that

$$
\lim _{t \rightarrow \alpha_{n}} t^{m(v+1)} K_{v+1}\left(2^{m} \mu_{n}^{1 / 2} t^{m} ; r-1 ; m\right)=0 .
$$

This completes the proof of Lemma 14. 
THEOREM 4. Suppose that for $\sigma>\sigma_{a}^{*}$

$$
\sup _{0 \leqq h \leqq 1}\left|\sum_{k^{2 m} \leqq \mu_{n} \leqq(k+h)^{2 m}} b(n) \mu_{n}^{\sigma-1 / 2 m}\right|=o(1),
$$

as $k \rightarrow \infty$. Then, (2.7) is valid for $q>2 m \sigma_{a}^{*}-m r-3 / 2$ and for those positive values of $x$ such that the left side of (2.7) is defined. The series on the right-hand side of (2.7) converges uniformly on any interval for $x>0$ where the left-hand side is continuous. The convergence is bounded on any interval $0<x_{1} \leqq x \leqq x_{2}$ when $q=0$.

Proof. Suppose the identity

$$
\begin{aligned}
& \frac{1}{\Gamma(\alpha+1)} \sum_{\lambda_{n} \leqq x}^{\prime} a(n)\left(x-\lambda_{n}\right)^{\alpha} \\
& \quad=2^{\alpha(1-m)} \sum b(n)\left(\frac{x}{\mu_{n}}\right)^{(r+\alpha) / 2} K_{r+\alpha}\left(2^{m}\left(\mu_{n} x\right)^{1 / 2} ; r-1 ; m\right)+Q_{\alpha}(x)
\end{aligned}
$$

is valid for some $\alpha$. Put $x=y^{m}$ and assume $y$ lies in an interval $J$ of length less than $\pi / m$. By Lemma $14, m 2^{m} y^{2 m-1}$ times the series on the right-hand side of (3.6) is uniformly equiconvergent on $J$ with the differentiated series of the Fourier series of a function with period $\pi / m$ which equals $\lambda(y) F_{r+\alpha+1}(y)$ on $I$, provided that $\alpha>2 m \sigma_{a}^{*}-m r-3 / 2$. But then, $\alpha+1>2 m \sigma_{a}^{*}-m r-1 / 2$. Hence, from (2.7)

$$
\begin{aligned}
2^{(\alpha+1)(1-m)} \lambda(y) F_{r+\alpha+1}(y)= & \frac{\lambda(y)}{\Gamma(\alpha+2)} \sum_{\lambda_{n} \leqq y^{2 m}}^{\prime} a(n)\left(y^{2 m}-\lambda_{n}\right)^{\alpha+1}-\lambda(y) Q_{\alpha+1}\left(y^{2 m}\right) \\
= & \frac{\lambda(y)}{\Gamma(\alpha+1)} \int_{0}^{y} \sum_{\lambda_{n} \leqq t 2 m}^{\prime} a(n)\left(t^{2 m}-\lambda_{n}\right)^{\alpha} 2 m t^{2 m-1} d t \\
& -\lambda(y) Q_{\alpha+1}\left(y^{2 m}\right) .
\end{aligned}
$$

Since $\lambda(y)=1$ on $J$, the theorem is now immediate from the localization principle and the properties of the Fourier series of

$$
\frac{2 m y^{2 m-1}}{\Gamma(\alpha+1)} \sum_{\lambda_{n} \leqq y^{2 m}}^{\prime} a(n)\left(y^{2 m}-\lambda_{n}\right)^{\alpha} .
$$

4. Examples. Chandrasekharan and Narasimhan have given several examples of identities when $m=1$. Consequently, we concentrate on $m>1$.

EXAMPLE 1. Consider $\zeta^{2}(2 s)=\sum d(n) n^{-2 s}$, where $d(n)$ denotes the number of divisors of $n$. Now, $\chi(s)=\Gamma^{2}(s) \pi^{-2 s} \zeta^{2}(2 s)$ has double poles at $s=0$ and $s=\frac{1}{2}$. After an elementary calculation, $(2.1)$ becomes

$$
\begin{aligned}
\sum d(n) E_{2}\left(\pi^{2} n^{2} y\right)= & y^{-1 / 2} \sum d(n) E_{2}\left(\pi^{2} n^{2} / y\right) \\
& -\frac{\gamma}{2}+\frac{1}{4} \log \frac{16 \pi^{2}}{y}+y^{-1 / 2}\left(\frac{\gamma}{2}-\frac{1}{4} \log 16 \pi^{2} y\right),
\end{aligned}
$$


where $\gamma$ denotes Euler's constant. Replacing $n$ by $n^{2}$, as $a(n)=0$ if $n$ is not a square, and $x$ by $x^{2}$, we have from Corollary 1 and Theorem 4 for $q>-\frac{1}{2}$,

$$
\begin{aligned}
\frac{1}{\Gamma(q+1)} \sum_{n \leqq x}^{\prime} d(n)\left(x^{2}-n^{2}\right)^{q} & \\
= & \frac{x^{2 q}}{4 \Gamma(q+1)}+\frac{\pi^{1 / 2} x^{2 q+1}}{4 \Gamma(q+3 / 2)}(2 \log x+\psi(1 / 2)-\psi(q+3 / 2)+4 \gamma) \\
& +\left(2 \pi^{2}\right)^{-q} \sum d(n)\left(\frac{x}{n}\right)^{q+1 / 2} \int_{0}^{\infty} u^{q} J_{-1 / 2}(u) J_{q+1 / 2}\left(4 \pi^{2} n x / u\right) d u,
\end{aligned}
$$

where the series on the right-hand side converges uniformly on any interval where the left-hand side is continuous and converges boundedly when $q=0$. Now, $J_{-1 / 2}(z)=(2 / \pi z)^{1 / 2} \cos z, J_{1 / 2}(z)=(2 / \pi z)^{1 / 2} \sin z$, and

$$
\int_{0}^{\infty} \cos u \sin \left(\frac{y^{2}}{u}\right) d u=-y\left(\frac{\pi}{2} Y_{1}(2 y)+K_{1}(2 y)\right),
$$

where $Y_{1}(z)$ and $K_{1}(z)$ are the Bessel functions usually so denoted. (4.2) is easily derived from formula (4) on p. 184 of Watson [13]. Thus, (4.1) for $q=0$ becomes

$$
\begin{aligned}
\sum_{n \leqq x}^{\prime} d(n)= & \frac{1}{4}+x(\log x-1+2 \gamma) \\
& -\sum d(n)\left(\frac{x}{n}\right)^{1 / 2}\left(Y_{1}\left(4 \pi(n x)^{1 / 2}\right)+\frac{2}{\pi} K_{1}\left(4 \pi(n x)^{1 / 2}\right)\right) .
\end{aligned}
$$

This last identity was first proven by Voronoi [12]. Proofs have also been given by Oppenheim [9] and Chandrasekharan and Narasimhan [4].

EXAmple 2. Consider $f^{2}(s)$ where $f(s)=\sum \tau(n) n^{-s}$. Since $\chi(s)$ is entire, (2.1) gives upon the substitution of $y / 4 \pi^{2}$ for $y$,

$$
\sum \tau^{\prime}(n) E_{2}(n y)=\left(4 \pi^{2} / y\right)^{12} \sum \tau^{\prime}(n) E_{2}\left(16 \pi^{4} n / y\right),
$$

where

$$
\tau^{\prime}(n)=\sum_{j k=n} \tau(j) \tau(k)
$$

By Corollary 1 and Theorem 4 we have for $q>1 / 2$

$\frac{1}{\Gamma(q+1)} \sum_{m n \leqq x} \tau(m) \tau(n)(x-m n)^{q}=\left(8 \pi^{2}\right)^{-q} \sum \tau^{\prime}(n)\left(\frac{x}{n}\right)^{6+q / 2} K_{12+q}\left(16 \pi^{2}(n x)^{1 / 2} ; 11 ; 2\right)$,

where the series on the right-hand side is uniformly convergent.

EXAMPLE 3. Let $K$ be an algebraic number field of degree $r_{1}+2 r_{2}$, where $r_{1}$ denotes the number of real conjugates in $K$ and $2 r_{2}$ the number of imaginary conjugates. The Dedekind zeta-function is defined by

$$
\zeta_{K}(s)=\sum F(n) n^{-s}
$$


where $F(n)$ denotes the number of nonzero integral ideals of norm $n$ in $K$. If $\Delta$ denotes the discriminant of $K, \zeta_{K}(s)$ satisfied the functional equation

$$
\xi(s)=\xi(1-s)
$$

where

$$
\xi(s)=\Gamma^{r}(s / 2) \Gamma^{r}(s) B^{-s} \zeta_{R}(s),
$$

where

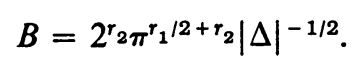

It is well known that $\zeta_{K}(s)$ is holomorphic except for a simple pole at $s=1$ with residue

$$
\lambda h=\frac{2^{r_{1}+r_{2}} \pi^{r_{2}} R h}{w|\Delta|^{1 / 2}}
$$

where $R$ is the regulator of $K, h$ the class number, and $w$ the number of roots of unity in $K$.

Suppose first that $r_{1}=0$. Then, $\zeta_{K}(s)$ satisfies Definition 1 with $\lambda_{n}=\mu_{n}=B n$ and $m=r_{2}$. From the functional equation it is easily seen that $\chi(s)=\Gamma^{r_{2}}(s) B^{-s} \zeta_{K}(s)$ is analytic except for poles at $s=0$ and $s=1$. Hence, the "modular" relation for $\zeta_{K}(s)$ becomes on replacing $y$ by $y / B$,

$$
\sum F(n) E_{r_{2}}(n y)=(B / y) \sum F(n) E_{r_{2}}\left(B^{2} n / y\right)+\zeta_{K_{2}^{2}}^{\left(r_{2}-1\right)}(0) /\left(r_{2}-1\right) !+\lambda h / y .
$$

From Theorems 2 and 4 for $q>r_{2}-3 / 2$ we find upon replacing $x$ by $B x$,

$$
\begin{aligned}
& \frac{1}{\Gamma(q+1)} \sum_{n \leqq x}^{\prime} F(n)(x-n)^{q} \\
& \quad=\frac{\lambda h x^{q+1}}{\Gamma(q+2)}+B^{-q} 2^{q\left(1-r_{2}\right)} \sum F(n)\left(\frac{x}{n}\right)^{(q+1) / 2} K_{q+1}\left(2^{r_{2}} B(n x)^{1 / 2} ; 0 ; r_{2}\right)
\end{aligned}
$$

If $r_{2} \geqq 2$, the series on the right-hand side converges uniformly for $q>r_{2}-3 / 2$. If $r_{2}=1$, the series converges uniformly on any interval where the left side is continuous, and boundedly on any interval $0<x_{1} \leqq x \leqq x_{2}<\infty$ when $q=0$. If $r_{2}=1$, i.e. if $K$ is an imaginary quadratic field, and $q=0$, the above identity is

$$
\sum_{n \leqq x}^{\prime} F(n)=\lambda h x+\sum F(n)\left(\frac{x}{n}\right)^{1 / 2} J_{1}\left(4 \pi(n x /|\Delta|)^{1 / 2}\right) .
$$

Suppose now that $r_{2}=0$. Then $\zeta_{K}(2 s)$ satisfies Definition 1 with $\lambda_{n}=\mu_{n}=B^{2} n^{2}$, $\chi(s)$ has poles at $s=0$ and $s=1 / 2$, and so (2.1) becomes with $y$ replaced by $y / B^{2}$

$$
\begin{aligned}
\sum F(n) E_{r_{1}}\left(n^{2} y\right)= & B y^{-1 / 2} \sum F(n) E_{r_{1}}\left(B^{4} n^{2} / y\right) \\
& +2^{r_{1}-1} \zeta_{K}^{\left(r_{1}-1\right)}(0) /\left(r_{1}-1\right) !+(1 / 2) \pi^{r_{1} / 2} \lambda h y^{-1 / 2} .
\end{aligned}
$$


For $r_{1}=1, \zeta_{K}(s)=\zeta(s)$ and the above formula reduces to the well-known thetarelation. Upon replacing $x$ by $B^{2} x^{2}$, we have from (2.7) for $q>r_{1} / 2-3 / 2$

$$
\begin{aligned}
& \frac{1}{\Gamma(q+1)} \sum_{n \leqq x}^{\prime} F(n)\left(x^{2}-n^{2}\right)^{q} \\
& \quad=\frac{\pi^{r_{1} / 2} \lambda h x^{2 q+1}}{2 \Gamma(q+3 / 2)}+B^{-2 q} 2^{q\left(1-r_{1}\right)} \sum F(n)\left(\frac{x}{n}\right)^{q+1 / 2} K_{q+1 / 2}\left(2^{r_{1}} B^{2} n x ;-1 / 2 ; r_{1}\right) .
\end{aligned}
$$

If $r_{1} \geqq 3$, the series on the right-hand side converges uniformly on any interval for $x>0$. If $r_{1}=2$, the series converges uniformly on any interval for $x>0$ where the left side is continuous, and converges boundedly on any interval $0<x_{1} \leqq x \leqq x_{2}<\infty$ if $q=0$. If $r_{1}=2$, i.e.if $K$ is a real quadratic field, the above identity for $q=0$ redfices to

$$
\sum_{n \leqq x}^{\prime} F(n)=\pi^{1 / 2} \lambda h x-\sum F(n)\left(\frac{x}{n}\right)^{1 / 2}\left[Y_{1}\left(4 \pi(n x /|\Delta|)^{1 / 2}\right)+\frac{2}{\pi} K_{1}\left(4 \pi(n x /|\Delta|)^{1 / 2}\right)\right] .
$$

\section{BIBLIOGRAPHY}

1. T. M. Apostol and Abe Sklar, The approximate functional equation of Hecke's Dirichlet series, Trans. Amer. Math. Soc. 86 (1957), 446-462.

2. S. Bochner, Some properties of modular relations, Ann. of Math. 53 (1951), 332-363.

3. K. Chandrasekharan and Raghavan Narasimhan, Hccke's functional equation and arithmetical identities, Ann. of Math. 74 (1961), 1-23.

4. - Functional equations with multiple gamma factors and the average order of arithmetical functions, Ann. of Math. 76 (1962), 93-136.

5. - The approximate functional equation for a class of zeta-functions, Math. Ann. 152 (1963), 30-64.

6. E. T. Copson, Theory of functions of a complex variable, Clarendon Press, Oxford, 1935.

7. Erich Hecke, Dirichlet series, Planographed Lecture Notes, Princeton Institute for Advanced Study, Edwards Brothers, Ann Arbor, Mich., 1938.

8. J. E. Littlewood, Lectures on the theory of functions, Oxford Univ. Press, New York, 1944.

9. A. Oppenheim, Some identities in the theory of numbers, Proc. London Math. Soc. (2) 26 (1927), 295-350.

10. Hans-Egon Richert, Uber Dirichletreihen mit Funktionalgleichung, Acad. Serbe Sci. Publ. Inst. Math. 11 (1957), 73-124.

11. E. C. Titchmarsh, Theory of Fourier integrals, 2nd ed., Clarendon Press, Oxford, 1948.

12. G. Voronoi, Sur une fonction transcendante et ses applications à la sommation de quelques séries, Ann. Sci. École Norm. Sup. (3) 21 (1904), 207-267, 459-533.

13. G. N. Watson, A treatise on the theory of Bessel functions, 2nd ed., Cambridge Univ. Press, Cambridge; Macmillan, New York, 1944.

14. A. Zygmund, On trigonometric integrals, Ann. of Math. 48 (1947), 393-440.

UNIVERSITY OF WISCONSIN, MADISON, WISCONSIN

UNIVERSITY OF GLASGOW, Glasgow, Scotland 